\title{
A case of preeclampsia developed massive ascites after delivery
}

\author{
Shota Higami $^{1}$, Emi Kondo ${ }^{1}$, Eiji Shibata ${ }^{1}$, Shigeki Fujimoto ${ }^{1}$, Marina Hagimoto ${ }^{1}$, Ruka \\ Urakawa $^{1}$, Tamaki Matsumiya ${ }^{1}$, Takayuki Uchimura ${ }^{1}$, Toshihide Sakuragi ${ }^{1}$, Shoko \\ Amimoto $^{1}$, Tomoichiro Kuwazuru ${ }^{1}$, Hiroshi Mori ${ }^{1}$, Satoshi Aramaki ${ }^{1}$, and Kiyoshi Yoshino ${ }^{1}$ \\ ${ }^{1}$ Affiliation not available
}

December 28, 2020

\begin{abstract}
Preeclampsia causes various presentations by increased endothelial permeability and microvascular damages. Maternal ascites related severe preeclampsia is generally explained by increased capillary permeability due to endothelial cell dysfunction and reduced intravascular oncotic pressure. Here we report a patient with postpartum massive ascites associated with preeclampsia.
\end{abstract}

\section{Title:}

A case of preeclampsia developed massive ascites after delivery

Authors:

Shota Higami, Emi Kondo, Eiji Shibata, Shigeki Fujimoto, Marina Hagimoto, Ruka Urakawa, Tamaki Matsumiya, Takayuki Uchimura, Toshihide Sakuragi, Shoko Amimoto, Tomoichiro Kuwazuru, Hiroshi Mori, Satoshi Aramaki, Kiyoshi Yoshino

\section{Affiliation of authors:}

Department of Obstetrics and Gynecology, University of Occupational and Environmental Health, Kitakyushu, Fukuoka, Japan

\section{Running title:}

Preeclampsia with massive ascites after delivery

\section{Corresponding author:}

Eiji Shibata, MD, $\mathrm{PhD}$

Department of Obstetrics and Gynecology, School of Medicine, University of Occupational and Environmental Health, Japan. Yahatanishi-ku, Kitakyushu 807-8555, Japan,

TEL: +81-93-603-1611

FAX: +81-93-691-9337

E-mail: age-s@med.uoeh-u.ac.jp

\section{Abstract:}

Preeclampsia causes various presentations by increased endothelial permeability and microvascular damages. Maternal ascites related severe preeclampsia is generally explained by increased capillary permeability due to endothelial cell dysfunction and reduced intravascular oncotic pressure ${ }^{1)}$. Here we report a patient with postpartum massive ascites associated preeclampsia. 
Key words: preeclampsia, ascites

\section{Key Clinical Message}

If abdominal distension is observed as in this case, it is necessary to suspect ascites retention and management carefully aggravation of $\mathrm{PE}$.

\section{Introduction:}

Preeclampsia (PE) is a syndrome caused by generalized endothelial dysfunctions. Although the underlying mechanisms of effusion into the body cavity in preeclampsia are controversial, pleural effusion and ascites in preeclampsia is generally explained by increased capillary permeability due to endothelial cell dysfunction and reduced intravascular oncotic pressure. Here we report a patient with postpartum massive ascites associated preeclampsia.

\section{Case report:}

A 26-year-old primigravid women was followed by an obstetrics practitioner over the course of a naturally conceived pregnancy. Proteinuria without hypertension was observed after 35 weeks of gestation. Ultrasonography (USG) did not detect ascites until 39 weeks of gestation. From 36 to 40 weeks of gestation the gained $7 \mathrm{~kg}$. At 40 weeks of gestation, she was admitted due to the premature rupture of the membrane. A vacuum delivery with Kristeller maneuver was performed because of fetal distress and maternal severe hypertension. The newborn was fetal growth restricted, weighing 2758 gram, with Apgar score of 8 and 9 at 1 and 5 minutes, and Umbilical $\mathrm{pH}$ of 7.084. Maternal blood pressure improved rapidly after delivery. No maternal abdominal symptoms were observed on the day of delivery. Two days after delivery, the mother exhibited increased abdominal volume. USG detected massive ascites, and she was transferred to our hospital.

Maternal blood pressure fluctuated around 140/80mmHg, with heart rate of 106bpm, and SpO2 99\% (room air) without respiratory distress. Massive ascites was confirmed by USG, Computed Tomography (CT) and abdominal puncture [Figure1]. Biochemical analysis revealed abnormal serum albumin $(1.8 \mathrm{~g} / \mathrm{dl})$ and creatinine $(0.97 \mathrm{mg} / \mathrm{dl})$ levels. Serum AST $/$ ALT was within normal limits and not applicable to HELLP syndrome. A spot urine sample protein and creatinine ratio was $1.19 \mathrm{~g} / \mathrm{g}$ Cre [Figure2].

Based on the above clinical condition, the admitting diagnosis was preeclampsia with massive ascites. If the patient had ascites due to preeclampsia, we expected to recover spontaneously after delivery. Blood pressure and blood creatinine levels recovered over a few days. The urine volume also increased and body weight and waist circumference decreased significantly. The patient was discharged on the seventh day of postpartum in good general condition. At the three month follow up, urine protein had improved to normal level and ascites was not detectable.

\section{Discussion:}

Preeclampsia is a life threating disorder with various symptoms. It is important to decide appropriate timing for delivery to prevent adverse outcome for mother and infant. There are several severe features which are key symptoms to decide this [Table1]. The pathophysiology of preeclampsia is increased vascular permeability and vascular spasm caused by endothelial dysfunction. Because effusion into the body cavity in preeclampsia is generally explained by increased capillary permeability due to endothelial cell dysfunction and reduced intravascular oncotic pressure, ascites is thought to be an early event in aggravated preeclampsia ${ }^{2)}$. Cong KJ et al. reported that the incidence of ascites in severe preeclampsia was 21.6/1000 during pregnancy ${ }^{33}$. On the other hand, Vaijyanath AM et al. estimated the incidence of ascites was 8 in 1000 during pregnancy and ascites usually develops from 27 to 31 weeks gestation in preeclampisa ${ }^{4)}$. They also reported that preeclampsia with massive ascites and respiratory distress due to pleural effusion during pregnancy should be signal pregnancy termination ${ }^{4}$.

In this case, massive ascites was observed after delivery. However, we may speculate that a certain level of ascites had accumulated before delivery based upon the $7 \mathrm{~kg}$ weight gain in the final month before delivery, 
and normal weight gain during the third trimester of pregnancy is about $0.5 \mathrm{~kg}$ per one week ${ }^{5)}$. Indeed, one described a relationship between ascites and rapid weight gain can occur in association with preeclampsia ${ }^{6)}$. Thus, it is necessary to anticipate the development or aggravation of preeclampsia when we see excessive weight gain during pregnancy.

The principal finding of this case is the acute retention of massive ascites occurring two days after delivery. Intra-abdominal hypertension is defined as a sustained intra-abdominal pressure (IAP) greater than $15 \mathrm{cmH} 20$. Antepartum IAP levels are thought to be significantly higher in patients with preeclampsia ${ }^{7)}$. The abdominal cavity can be considered as a semi-closed compartment, and any volume change in abdominal content can affect IAP.

In the present case, we speculate that the IAP level was elevated with the development of PE and existence of ascites before delivery. After delivery IAP decreased significantly because of a sudden involvement of the uterus which occupied most of the abdominal cavity. Finally, according to rapid decreased IAP levels at the time of delivery, a large amount of ascites leaked into the abdominal cavity. Thus, massive ascites was detected postpartum.

The level of soluble fms-like tyrosine kinase-1 (sFlt-1) in this case was higher in postpartum period. There is no diagnostic criteria for sFlt-1 values, but current reports describe the level of sFlt-1 as gradually decreasing after delivery and stabilized around $77 \mathrm{pg} / \mathrm{ml}$. In this case, it was suggested that sFlt-1 was high at the time of delivery ${ }^{8)}$. sFlt-1 is known as important factor for microvascular damage. The model suggests elevated sFlt-1 and associated microvascular damage may be severe during delivery and ascites was likely to accumulate.

There are several reports relating PE with massive ascites. One cohort study reported that ascites was associated with maternal events such as eclampsia, pulmonary edema, renal failure, and disseminated intravascular coagulation ${ }^{9)}$. A secondd prospective cohort study showed an association between ascites and both maternal and perinatal outcomes such as premature obstetrics and low birth weight ${ }^{10)}$. Although both studies described patients diagnosed with severe PE, the case described here did not show severe hypertension nor any other related pathological features. However, this case was associated with FGR and neonatal distress. Thus, the accumulation of ascites itself may indicate that severe PE and/or aggravation of PE.

There is no clear evidence for management of PE associated with ascites. According to one report, early termination may improve the outcome in cases of preeclampsia associated with ascites ${ }^{11}$. Moreover, another report showed that development of ascites in preeclampsia warrants immediate termination of pregnancy in a study of 21 patients with pregnancy induced hypertension. Based on the existing literature and this case, earlier termination should be considered to improve both maternal and infant outcomes if obvious ascites is observed with preeclampsia.

In addition, critical actions, such as albumin replacement therapy or abdominal puncture to remove ascites, may be required if serious complications including respiratory distress accompany massive ascites in postpartum. In this case, massive ascites decreased naturally as preeclampsia improved, and no medical intervention was needed for the management of massive ascites.

\section{Conclusion:}

Massive ascites may occur after delivery associated with decreased level of IAP in PE. Furthermore, if excessive weight gain or ascites have been detected in pregnant women with preeclampsia, we should consider their condition as severe PE or rapid aggravation of PE.

\section{Consent:}

Declaration of patient consent

\section{Acknowledgment:}

This work was supported by our institution. 


\section{Author Contribution:}

SH, EK, ES, TU, TS, SA, TK, HM, and SA were actively involved in the clinical care patients. SF, MH, RU and TM searched the literatures. SH, EK and ES wrote the manuscript. KY revised the manuscript.

\section{Conflict of interest:}

The authors declare no conflict of interest.

\section{References:}

1. Brown MA, Zammit VC, Lowe SA. 1989. Capillary permeability and extracellular fluid volumes in pregnancy-induced hypertension. Clin Sci (Lond) 77:599-604.

2. Dah CD, SenzenHsu, Her YS, Ta WC. 2005. Massive Ascites in Severe Pre-Eclampsia. Taiwanese Journal of Obstetrics and Gynecology 44:384-386

3. Cong KJ, Wang TT. 1994. Complication of ascites in pregnancy-induced hypertension. Zhonghua Fu Chan Ke Za Zhi 29:7-58.

4. Vaijyanath AM, Nayar B, Malhotra N, Deka D. 2002. Massive ascites in severe pre-eclampsia: a rare complication. J Obstet Gynaecol Res . 28:199-202.

5. Rasmussen KM, Yaktine AL. 2009. Institute of Medicine (US) and National Research Council (US) Committee to Reexamine IOM Pregnancy Weight Guidelines, eds. Weight Gain During Pregnancy: Reexamining the Guidelines . Washington (DC): National Academies Press (US).

6. American College of Obstetricians and Gynecologists; Task Force on Hypertension in Pregnancy. 2013. Hypertension in pregnancy. Report of the American College of Obstetricians and Gynecologists' Task Force on Hypertension in Pregnancy. Obstet Gynecol 122:1122-1131.

7. Mesut A. Ünsal, Ülkü İnce, Sevil Cengiz, S. Caner Karahan, Turhan Aran. 2017. The Relationship Between Intraabdominal Hypertension and Preeclampsia. Gynecology and obstetrics and reproductive medicine 23:1-5

8. Saleh L, van den Meiracker AH, Geensen R, et al. 2018. Soluble fms-like tyrosine kinase-1 and placental growth factor kinetics during and after pregnancy in women with suspected or confirmed pre-eclampsia. Ultrasound Obstet Gynecol 51:751-757.

9. J. Yavana Suriya, Anish Keepanasseril, K. Manikandan. 2017. Maternal ascites an independent prognostic factor in severe preeclampsia: a matched cohort study. Archives of Gynecology and Obstetrics 296:63-68.

10. Mbonyizina C, Ntirushwa D, Bazzett-Matabele L, Ntasumbumuyange D, Rulisa S, Magriples U. 2019. Point of care ultrasound: does the presence of ascites in severe pre-eclampsia correlate with poor maternal and neonatal outcome?. Trop Med Int Health24:1018-1022.

11. Woo JS, Liang ST, Wong VT. 1982. Ultrasonic detection of maternal ascites in patients with severe pre-eclampsia. Aust N Z J Obstet Gynaecol 22:237-239.

\section{Hosted file}

Figure12020.12.7.pdf available at https://authorea.com/users/385818/articles/501051-a-caseof-preeclampsia-developed-massive-ascites-after-delivery

\section{Hosted file}

Table1 2020.12.7.xlsx available at https://authorea.com/users/385818/articles/501051-a-caseof-preeclampsia-developed-massive-ascites-after-delivery

\section{Hosted file}

Table2 Laboratory data 2020.12.7.xlsx available at https://authorea.com/users/385818/ articles/501051-a-case-of-preeclampsia-developed-massive-ascites-after-delivery 